\title{
Integrated Sensor Wireless System for Alternative Energy Applications
}

\author{
Maher Rizkalla, Mohamed El-Sharkawy, and Penghua Sun
}

\begin{abstract}
These Wind turbines convert mechanical energy into electrical energy for storage and consumption. Pressure transducers are important in reflecting pressures within the hydraulic cylinders; they can provide continuous and reliable operation that enhances the reliability and efficiency of the overall system. Monitoring high temperature fluctuation will be necessary for the resolution and efficiency of the system. An integrated sensor system should be important for monitoring the tower vibration for bending, crack formation, and wave vibration.

Smart nanotechnology materials have been recently utilized in sensing applications. Carbon nanotube (CNT) based SoC sensor systems have potential applications in various fields, including medical, energy, consumer electronics, computers, and HVAC (heating, ventilation, and air conditioning), among others.

In this study, a nanotechnology multisensory system was designed and simulated using Labview Software. More emphasis are given to both pressure and temperature sensors that serve as transducers for the power mills. The mathematical models were developed for sensing three physical quantities: temperature, gas, and pressure. Four CNT groups on a chip (two for gas sensor, one for temperature, and a $4^{\text {th }}$ one for pressure) were utilized in order to perform sensing multiple parameters. The proposed fabrication processes and the materials used were chosen to avoid the interference of these parameters on each other when detecting one of them. The simulation results were translated into analog voltage from Labview software, transmitted via Bluetooth network, and received on desktop computers within the vicinity of the sensor system. The mathematical models and simulation results showed as high as $\mathbf{9 5 \%}$ accuracy in measuring temperature, and the $5 \%$ error was caused from the interference of the surrounding gas. Within $7 \%$ change in pressure was impacted by both temperature and gas interference.
\end{abstract}

Index Terms-Wind energy, nanotechnology, pressure sensors, temperature sensors, SOC.

\section{INTRODUCTION}

Wind power is the fastest growing energy resource with a capacity exceeded 120GW [1]. Research efforts have been focused on producing high quantity and quality power generation and overcoming the failure of wind turbines. Monitoring systems by analyzing data collection from wind turbine components, gearboxes, shafts, and the yaw systems, are necessary for early fault detection. Frequent DSP based maintenance may enhance the efficiency and avoid future faults from occurrences. Furthermore, machine conditions

Manuscript received January 10, 2015; revised May 18, 2015.

The authors are with the Department of Electrical and Computer Engineering, Purdue School of Engineering and Technology, Indiana University Purdue University Indianapolis (IUPUI), 723 W Michigan Street, Indianapolis, IN 46202 (e-mail: mrizkall@iupui.edu, melshark@iupui.edu). and accuracy are key factors for successful economical plans, and this is a driving force for research development, tackling fault issues for better efficiency and higher reliability.

There have been plenty of efforts in recent years exploring diagnostic methods in order to detect faults within windmill systems such as rotor fault, short winding fault, air gap fault, bearing fault, load fault, etc. When a fault is present, the frequency spectrum of the line current changes, leading to air gap modulation and resulting in rotating frequency harmonics in the machine inductances. Approaches for detection therefore include electromagnetic field, temperature change, mechanical vibration, faulty currents, etc. Signal processing tools such as wavelet transformation and artificial intelligence have been proposed for fault diagnoses and detections within motors. In some cases, these tools cannot provide comprehensive fault coverage with high reliability. It is important to detect early faults before it is developed. Early fault detection by continuous monitoring may avoid catastrophic costly future failures, leading to safe operating environment.

In order to maximize the utilization of the wind machine and minimize the maintenance overhead cost, a wireless network system that is capable of continuous monitoring of windmill system must be in place. This eliminates installation cost and continuous maintenance. This may provide information regarding the health condition of the machines in order to prevent future mechanical fault failures. High sensitivity sensor system may be required to detect small fraction or bending via minor change in vibration. Accelerometers are very limited to detect this change.

In this work, a novel approach in emerging nanotechnology into renewable energy by designing an integrated sensor system that is capable to detect small changes in multiple parameters such as bending, fracture, and temperature variation. Nanotechnology smart materials possess high sensitivity that can detect small cracks for instance as soon as they occur. Therefore, the use of nanotechnology materials and devices within a SoC (system on Chip) should enhance the diagnostic capability by detecting small change above a threshold value. The slightly change in temperature may occur from power dissipation of faulty currents.

\section{A. Wind Turbine and Need of Transducers}

Pressure-temperature sensors are necessary for the windmill applications. They should be rugged and capable of operating over a wide range of temperatures with good electromagnetic shielding protection. The feedback stability with pressure and temperature sensors should maintain the level of oil to keep appropriate supply of power energy from the windmill.

The windmill is a machine that converts wind energy into 
electricity. The wind turbine components are shown in Fig. 1. Sensors are critical components in operation due to the desert areas where these windmills function. The maintenance of these windmills is important for the efficient system. Temperature sensors are also important to control for proper operation. Operating temperature for these windmills may range from -50 to $60^{\circ} \mathrm{C}$. For maximum wind turbine efficiency, the wind towers should be installed in areas of steady winds. The turbines must also be aligned with the winds in order to maximize the power generation from the mechanical energy. Proper alignment may be controlled through the use of wind controllers that are often powered and monitored by pressure transducers that convert the mechanical energy into electrical energy for control processing.

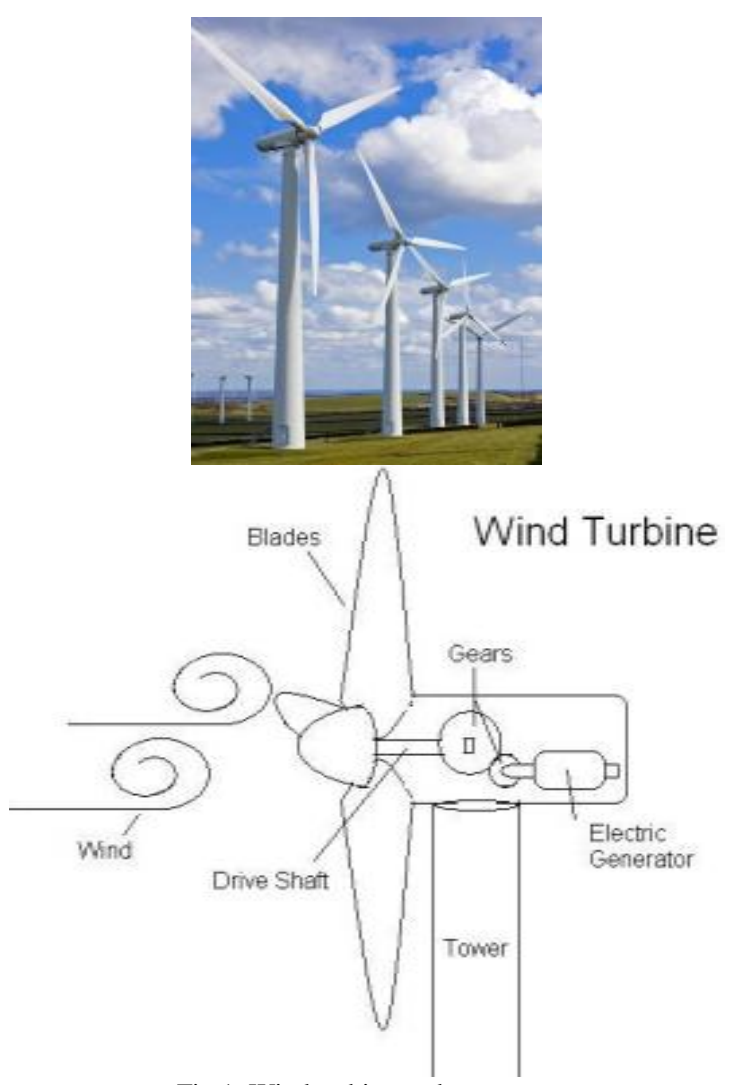

Fig 1. Wind turbine and components.

\section{B. Nanosensor Devices}

Nanotechnology has recently explored unique features related to smart nanomaterials such as fullerene, carbon nanotube, graphene, quantum dots, nanophotonic crystals, magneto resistance material, and nano polymers. Fig. 2 gives the image of the Fullerene, quantum dots and CNTs, that can sense physical quantities such as temperature, pressure, gas, and magnetic fields by changing their electrical or magnetic properties. They can change their conductivity when exposed to a gas. The capacitance of the device will also change when the CNTs are exposed to these physical quantities. There are mainly three types of CNTs: Single-walled (SWCNT), double-walled (DWCNT) and multi-walled (MWCNT). Single-walled CNTs can be seen as a closed-loop of graphene (diameter of about 1 nanometer), while double-walled and multi-walled CNTs are basically several single-walled ones placed in a coaxial configuration (diameter of about 10 s of nanometers). Reference [2] discussed the design of a radio frequency (RF) based system that detected the change in the reflected RF power due to the change in the device capacitance, and accordingly, to the exposed gas. The fabrication of the CNTs may be demonstrated by separation and enrichment.

Researchers have succeeded to implement nanotechnology sensors that sense one parameter such as temperature, gas, or pressure [3], [4]. There is a need, however, to have multiple sensors on a chip that can detect a combination of parameters simultaneously to perform multiple bio functions. Since the CNT material changes with all parameters when exist simultaneously, it is hard to detect the impact of each parameter on the CNT based sensor. Several examples include the exposure of the CNT device to both temperature and gas present in the same chamber. The resulting change in the resistance cannot precisely predict the change of each, separately. Furthermore, the change in the pressure in the presence of the temperature and gas may not reflect the true value of the pressure quantity. A reliable system should resolve the interference in order to separately detect the impact of every parameter separately. Therefore, building three sensors in the same chip, each to detect one quantity separately is quite challenging since the three sensors are set into the same chip and exposed to all parameters' changes. Other issues may include the type of assembly of the CNT materials that may be chosen to provide the range of temperature, gas, or pressure to be suitable for Bio-applications

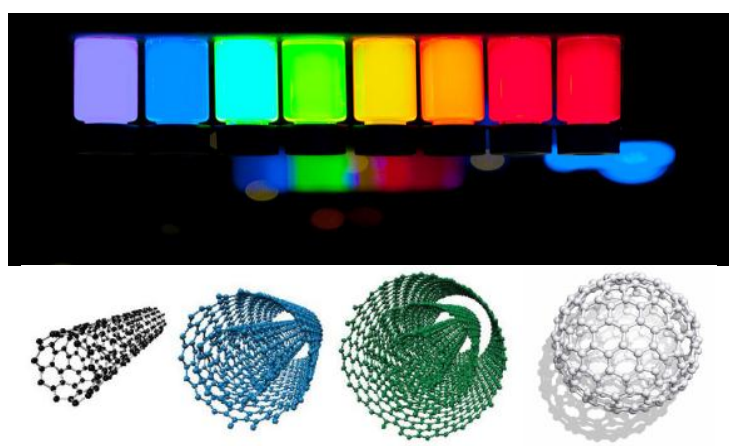

Fig. 2. Images of quantum dots, CNTs, and fullerene.

\section{The Processing Unit}
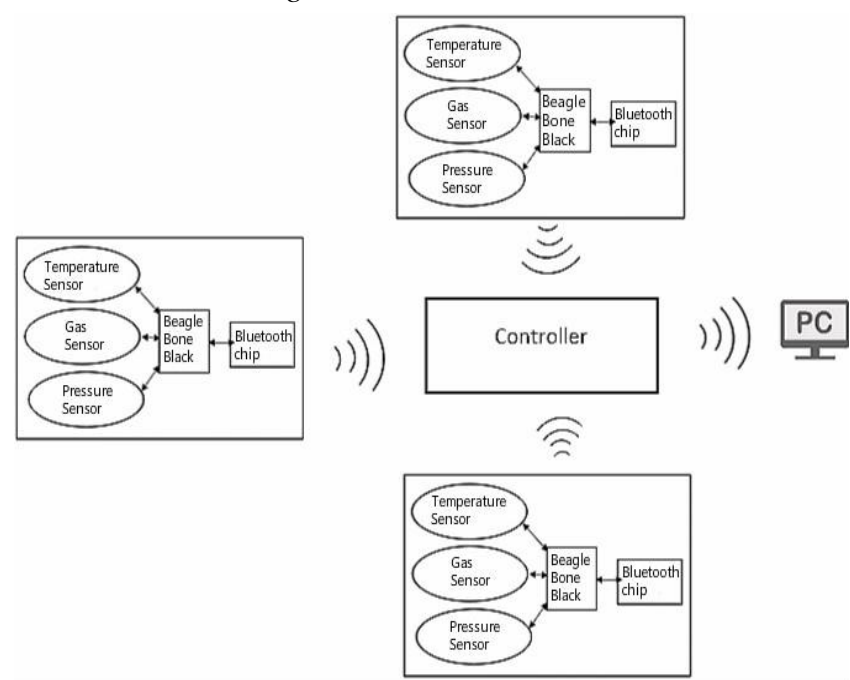

Fig. 3. The system diagram. 
The temperature and pressure sensor system may be utilized in bioengineering applications, including electrocardiography (ECG), human temperature, and heart rate, among others. Fig. 3 shows a general medical setting for the use of multiple sensor system via wireless network.

\section{INTEGRATED SYSTEM MODEL}

\section{A. The Pressure Sensor}

The pressure sensor designed for this system was based on detecting the change in conductivity caused by pressure, resulting in a change of the material resistance. The substrate material selected for this purpose was chosen based on its linearity and pressure coefficients. PMMA (polymethyl methacrylate is a versatile polymeric material that is well suited for many imaging and non-imaging microelectronic applications. It is a common positive resist for e-beam, X-ray, and deep UV micro lithographic processes) substrate material was found to be appropriate for this design [5]. Fig. 4 gives schematics of the pressure sensor. Fig. 5 gives the basis of the mathematical model for the analysis. The detection of a clamped circular shape under a uniform pressure $P$ is denoted by $w$ and is given by [6]:

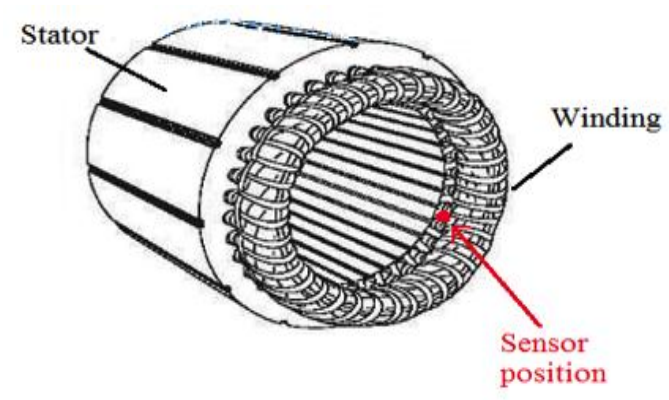

Fig. 4. Suggested integrated sensor location.

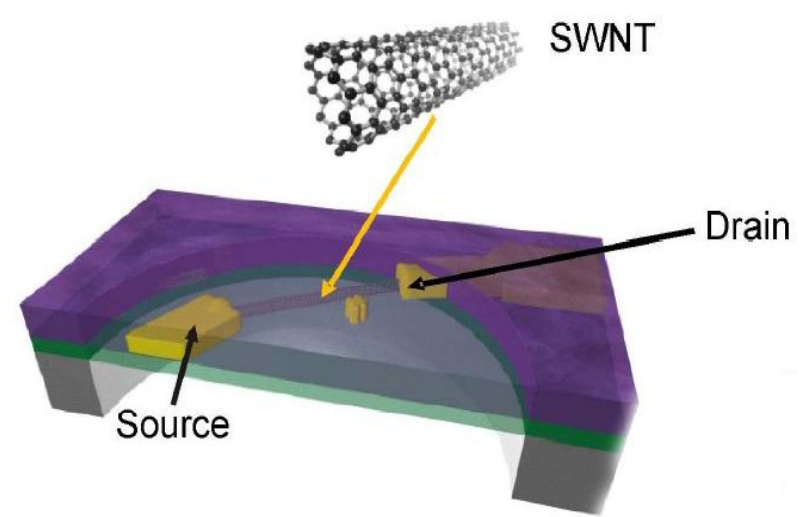

Fig. 5. The pressure sensor.

Based on Fig. 6 below,

$$
w=\frac{p a^{2}}{264 D}\left[1-(r / a)^{2}\right]^{2}
$$

where $r$ and $a$ are the radial coordinate and diaphragm radius, respectively. It is clear from that $r$ is the original radius and $a$ is the radius change. $D$ is a measurement of stiffness and is given by:

$$
D=\frac{E h^{3}}{12\left(1-v^{2}\right)}
$$

where $E, h$, and $v$ are Young's modulus, plate thickness, and Poisson's ratio, respectively.

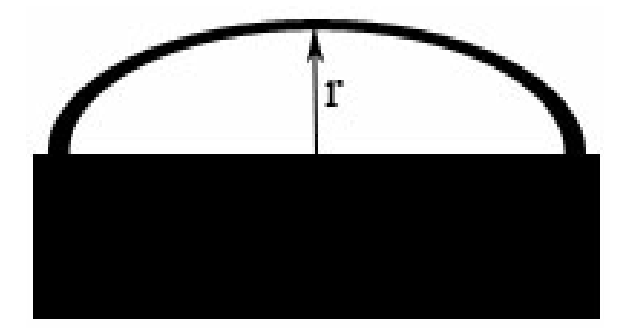

Fig. 6. The shape of PMMA device.

The room temperature resistance at $0 \mathrm{kPa}$ of a sensor was typically ranged from several $\mathrm{k} \Omega$ to several hundred $\mathrm{k} \Omega$. Some works have been done previously [7] showed that the resistance across the CNTs increases linearly with applied pressure up to $70 \mathrm{kPa}$. Fig. 6 gives the pressure-detection curve, showing the effect of the diameter size of the substrate.

\section{B. The Resistance Model}

When you submit your final version, after your paper has been accepted, prepare it in two-column format, including figures and tables.

The gauge factor of the CNT is given by [8]:

$$
G=\frac{\Delta R}{R}\left(\frac{1}{\varepsilon}\right)
$$

where $R$ and $\Delta R$ are the initial resistance of the sensor before the pressure is applied, and the resistance change of the CNT under the pressure, respectively, and $\varepsilon$ is the strain of the sensor material. The resistance as function of the strain parameter is given by [8]:

$$
R(\varepsilon)=R_{0}+\tilde{R}_{1} \varepsilon
$$

In this expression, $R_{0}$ is the resistance without strain; $\widetilde{R}_{1}$ is the strain coefficient. The model with the strain coefficient is given by [6]

$$
\begin{gathered}
R(\varepsilon)=R_{S}+\frac{1}{|t|^{2}} \frac{h}{8 e^{2}}\left[1+\exp \left(\frac{\tilde{E}_{g} \varepsilon}{k_{B} T}\right)\right] \approx \\
\left(R_{S}+\frac{1}{|t|^{2}} \frac{h}{4 e^{2}}\right)+\left(\frac{\tilde{E}_{g}}{|t|^{2}} \frac{h}{8 e^{2} k_{B} T}\right) \varepsilon
\end{gathered}
$$

where $R_{S}$ is the series resistance of the junction due to SWCNT metal contacts, $|t|^{2}$ is the transmission through the nanotube, and $E_{g}=\tilde{E}_{g} \varepsilon$ is the strain-dependent band gap for metallic nanotubes, neglecting the torsion contributions. Fig. 7 shows a typical resistance-stain curve, showing the effect of the diameter size in the pressure range, leading to the proper deflection that may be detected by the processing unit.

The sensitivity of CNT to detect pressure changes was found to be $54 \mathrm{pA} / \mathrm{mbar}$ for three different strain coefficients averages [5], [9]. Fig. 8 shows the sensitivity curve considered for the design of the pressure sensor. Heartbeat pressure is about $170 \mathrm{mbar}$, and the current change is about $10 \mathrm{nA}$ current change $(\mathrm{Vds}=200 \mathrm{mV}, \mathrm{Vg}=4 \mathrm{~V})$, which make the design of the sensor system appropriate for bio-engineering applications. This shows the appropriate sensitivity of the selected material. 

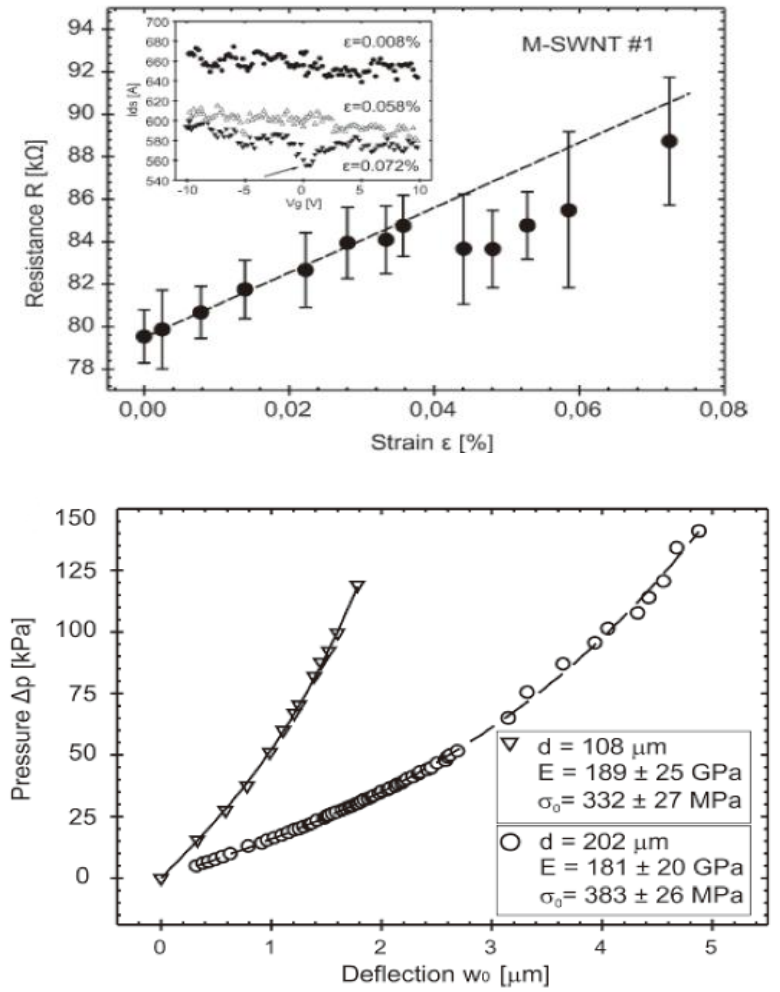

Fig. 7. The pressure/deflection and strain curves.

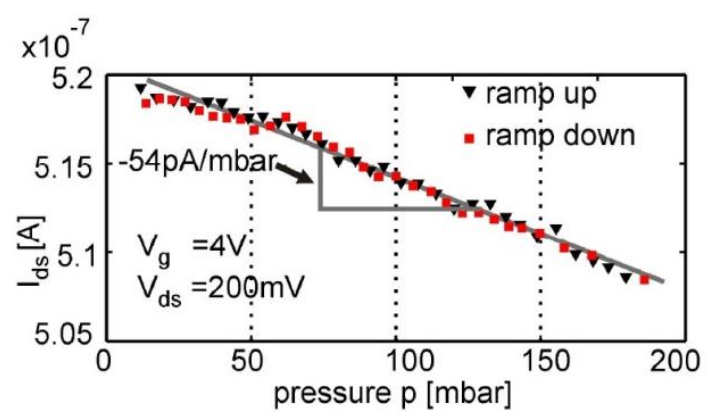

Fig. 8. The sensitivity curve of pressure sensor.

As it can be seen from the data information of this sensor, the sensor is appropriate to measure up to 200 mbar linear range. The minimum current observed was near 200 mbar at $0.5 \mu \mathrm{A}$, which is still appropriate to be processed by the hardware-processing unit.

\section{The Temperature Sensor Model}

The concept of the temperature sensor is based on exhibiting the change in the CNT resistance material when exposed to change in temperature. The temperature change may also alter the pressure, and the thermal expansion (or contraction) of the Polyamide material.

The traditional approach by placing the CNT within a MOSFET device may not be appropriate for a multisystem SOC approach. This work proposes two expansion elements of a material called polyimide for the temperature sensing elements. There are CNTs surrounding the polyimide to detect the pressure change. Thus, we can calculate the temperature. Fig. 9 shows the structure of sensor. The two expanding elements are placed at a certain level. When the temperature gets higher, the expanding elements expand and give more pressure to the CNT, causing an increase to the CNT resistance. While, when the temperature goes down, the expanding elements shrink and the CNT's resistance becomes smaller.

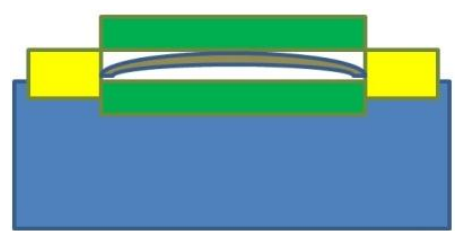

Fig. 9. The temperature sensor

When the temperature changes, the polyamide alters its volume, and accordingly, the pressure from it to the CNTs sensing material. Then the CNTs will exhibit a change in resistance that can be detected, and the change will be processed by the DSP unit, producing the pressure data in the form of electrical energy. The model of the pressure sensing element of the temperature sensor is the same as the pressure sensor model. Thermal stress pressure can be written as $\Delta P=E \alpha \Delta T$, Where $E$ is young's modulus which is near 1.1 $\mathrm{GPa}$ for polyamide, and $\alpha$ is the thermal expansion coefficient and it is about $110 \times 10^{-6} \mathrm{~m} / \mathrm{mK}$ for the same material.

\section{THE WIRELESS TRANSMISSION}

If you are using Word, use either the Microsoft Equation Editor or the MathType add-on (http://www.mathtype.com) for equations in your paper (Insert | Object | Create New | Microsoft Equation or MathType Equation). "Float over text" should not be selected.

In this section, the complete wireless transmission based on BBB (developed by N.I.) and the Bluetooth techniques were introduced.

\section{A. Hardware Board System}

The assembly of the hardware system consists of three main components: BBB board, Bluetooth chip, and the control unit. The micro USB port of BBB simply works as a power source port; the micro HDMI port was linked to a monitor; the micro SD card was attached to the reader, which worked as a hard disk to the system. The standard USB port was interfaced to the USB hub and then attached to the USB based Bluetooth chip, and the mouse and keyboard adapter. Fig. 10 shows the BBB board used in this work. In this application, the BBB board functions as a mini PC that communicates with the Bluetooth system. It receives the data from the Labview software and transmits it to another Bluetooth device.

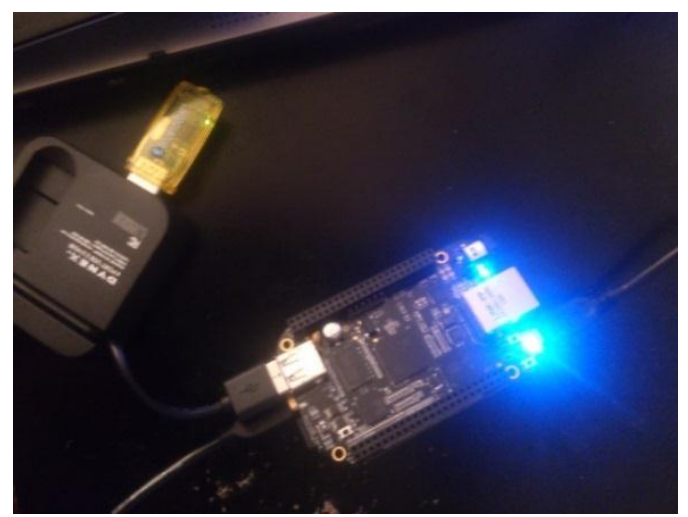

Fig. 10. Connection diagram of BBB board. 


\section{B. Results and Discussions}

In this section, we report simulation results from Labview for the integrated sensor system, including the temperature, gas, and pressure sensors. The wireless transmission for the dynamic responses of the sensors was verified. The simulation here presents data for one sensor as sample data for the multisensory system.

\section{The Temperature Sensor}

The range of the temperature used for testing the sensor was from 283.15 to $353.15 \mathrm{~K}$, which corresponds to 10 and $80^{\circ} \mathrm{C}$, respectively. This range was chosen in order to perform linear response with the CNT resistance. Fig. 11 shows the details of the response following the mathematical model. As it can be seen, the curve fits linearly well within the temperature range of 283 to $353 \mathrm{~K}$, and beyond this range, the response is not linear. This is the useful range of Bio and HVAC applications. The $x$ axles are time (s). The resistance is changing from 75 to $700 \mathrm{k} \Omega$ and the sensitivity range of the CNT pressure sensing part is $0 \sim 8400 \mathrm{kPa}$. Fig. 11 describes the resistance-temperature direct relationship. The slope of the curve (within the desired range of temperature) gives the sensitivity determined by $\Delta R / \Delta T$. In this case, the sensitivity is $0.7 \mathrm{k} \Omega / \mathrm{K}$. This indicates that the sensor can detect as small as milli $\mathrm{K}$ change, since the corresponding change in resistance is still in the $10 \mathrm{~s} \mathrm{k} \Omega$ range, and this can be easily detected by the processing unit of the system.
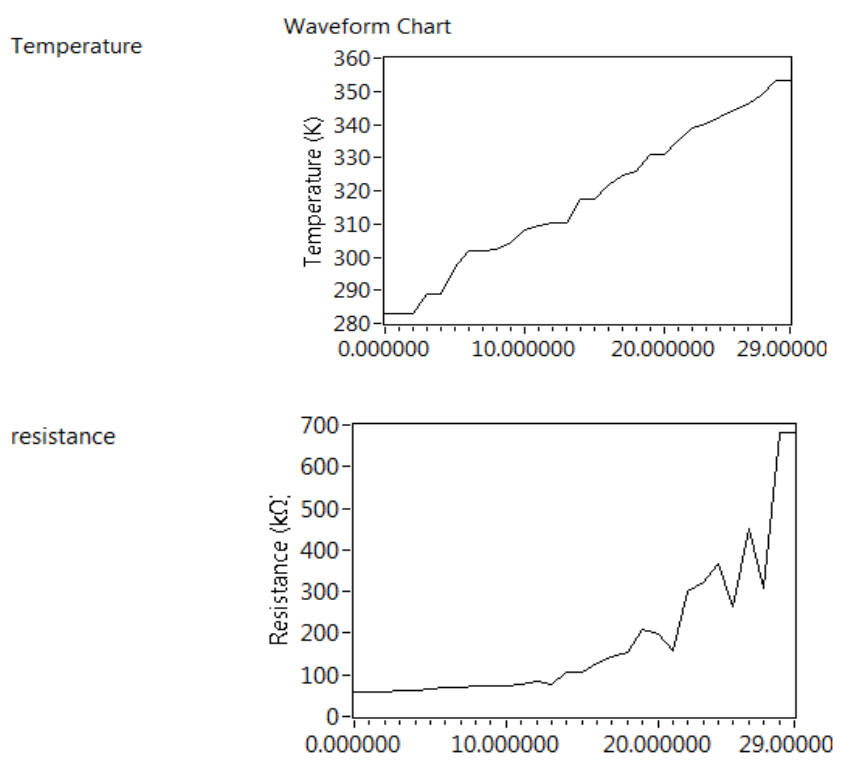

Fig. 11. The simulation result of the temperature sensor.

The design of the integrated sensor system built on the same chip, detecting temperature, gases, and pressure that interfere with each other is demonstrated. The nanodevices utilized CNT smart nanomaterials that are appropriate for bio-engineering applications as evidenced by the power level have been determined. The range of detection is determined from the linear part of the sensor characteristics. The error factor from interference was compensated within the DSP unit. The proposed system can be expanded to include more sensors such as liquid and gas flow or blood sugar detectors. For the temperature sensor, an indirect detection method was introduced. The CNTs worked as the pressure sensor to detect the volume change of the thermal expansion elements to avoid the gas influence. In this case, a read-out circuit was to be interfaced to the sensor in order to process the data.

For the gas sensor, the CNTs coating with SS-DNA device was given. The two sequences of SS-DNA made the CNTs significantly more sensitive to the gas. To raise the accuracy of the sensor, the twin-sensor with both sequence 1 and sequence 2 SS-DNA coated CNTs was used. The DSP chip could be modified to incorporate both sequence 1 and sequence $2 \% \Delta \mathrm{I} / \mathrm{I}$ data, into the table look up. For the pressure sensor, the CNTs placed between source and drain of the CMOS device was introduced. To reducing the effect of the temperature and gas, the CNTs were coated with Parylene C material.

\section{CONCLUSION}

This paper presents a general approach using SOC based integrated sensor system that can fit in many applications, including renewable energy, medical devices, HVAC, among others. The system features high sensitivity to small differential temperature or pressure changes. The change in temperature could be related to short circuit current dissipation, while the pressure sensor may monitor mechanical vibration or bending at its early stage.

The work presented here is based on simulation and device modeling. It brings emerging nanotechnology into renewable energy field. Other approaches using embedded sensor systems with I2C communication, with internet of things for wireless system is reserved for future consideration.

\section{ACKNOWLEDGMENT}

The authors would like to offer their appreciations for Dr. Euzeli Dos Santos for his assistance with preparing this manuscript.

\section{REFERENCES}

[1] H. Z. Huang, K. J. Zhang, and Y. Zhang, "Detection of turbine generator field winding serious inter-turn short circuit based on the rotor vibration feature," in Proc. the 44th International IEEE Universities Power Engineering Conference, Sept. 1-4. 2009, pp. 1-5, Glasgow, Scotland.

[2] B. Fitzgerald, J. Arrigan, and B. Basu, "Damage detection in wind turbine blades using time-frequency analysis of vibration signals," in Proc. the 2010 International Joint Conference on Neural Networks, July 18-23, 2010, pp. 1-5, Barcelona, Spain.

[3] N. K. Kumawat et al., "MEMS based motor fault detection in windmill using neural networks," International Journal of Research in Engineering and Science, vol. 2, no. 4, pp. 53-57, 2014.

[4] M. Fujioka, H. Watanabe, Y. Martin, M. Nakano, "Separation and enrichment of semiconducting carbon nanotubes and its application to highly sensitive carbon nanotube gas sensor," in Proc. 2011 IEEE Nanotechnology Materials and Devices Conference, October 18-21, 2011, Jeju, South Korea.

[5] R. Verma, K. Said, J. Salim, E. Kimathi, M. Rizkalla, S. Shrestha, M Agarwal, and K. Varahramyan, "Carbon nanotube-based microstrip antenna gas sensor," in Proc. 2013 IEEE 56 ${ }^{\text {th }}$ International Midwest Symposium on Circuits and Systems, Aug. 7-9, 2013, Columbus, Ohio.

[6] C. Y. Kuo, C. L. Chan, C. Gau, C.-W. Liu, S. H. Shiau, and J.-H. Ting, "Nano temperature sensor using selective lateral growth of carbon nanotube between electrodes," IEEE Transactions on Nanotechnology, vol. 6, no. 1, January 2007

[7] C. K. M. Fung, M. Q. H. Zhang, R. H. M Chan, and W. J. Li, "A Pmma-basedm Icrop Ressure Sensor Chip Using Carbon Nanotubeasssensingel Ements," Centre for Micro and Nano Systems, The Chinese University of Hong Kong, Hong Kong SAR.

[8] B. Crawford, D. Esposito, D. Pelletier, and V. Jain, "Flexible carbon nanotube based temperature sensor for ultra-small-site applications," 
Mechanical Engineering Undergraduate Capstone Projects, Northeastern University.

[9] S. G. Zhou and M. Hara, "Fabrication of a carbon nanotube-based gas sensor using dieiectrophoresis and its application for ammonia gas sensor using dieiectrophoresis and its application for ammonia detection by impedance spectroscopy," Journal of Physics D., vol. 36 , pp. 109-114, 2003.

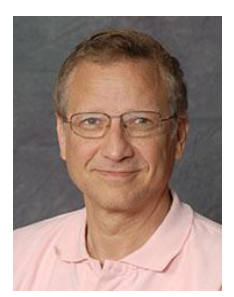

Maher Rizkalla was born in Assiut, Egypt on September 16, 1952. He received his Bs.EE degree from Assiut University and Ms.ECE degree from Cairo University in 1975 and 1980 respectively. He received his Ph.D. degree from Case Western Reserve University, Cleveland, Ohio in January 1985, all in electrical engineering. From January 1985 till August 1986, he was a research scientist at Argonne National Laboratory, Argonne, IL, while working as a visiting assistant professor at Purdue University Calumet. In August 1986, he joined the ECE Department at Purdue University Indianapolis where he is now a professor and the associate chair of the department.

His research interests include solid-state semiconductor and nanotechnology devices, electromagnetics, VLSI design, and applied superconductivity, with emphasis on MEMS/NEMS applications. Dr. Rizkalla published more than 175 papers in these areas, and received a number of governmental and industrial grants and contracts, among those four from NSF and two from Department of Education. He supervised more than 40 graduate students.
He received the Professor of the Year Award from Purdue University Calumet in 1986, and five times of the Best Teaching Awards from IUPUI $\mathrm{He}$ is a senior member of IEEE, and a professional engineer registered in the State of Indiana.

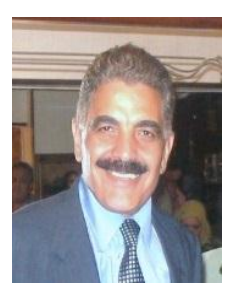

Mohamed El-Sharkawy has over thirty years of research and teaching experience in the areas of digital signal processing and communications. He wrote four books and over two hundred papers. He received several million dollars of research grants from industrial partners. He spent two sabbatical years at Motorola Inc. and FreeScale Inc. He is the principal thesis advisor for over 60 graduate students.

$\mathrm{He}$ received the Outstanding Graduate Student Award from Southern Methodist University in 1985. He received the Abraham M. Max Distinguished Professor Award from Purdue University in 1996. He received the US Fulbright Scholar Award in 2008. He received the Prestigious External Award Recognition Award from Purdue School of Engineering and Technology, 2009. He is a reviewer for the National Science Foundation and Fulbright. 